\title{
Insônia: prevalência e fatores de risco relacionados em população de idosos acompanhados em ambulatório
}

\author{
Insomnia: prevalence and related risk factors in elderly outpatients
}

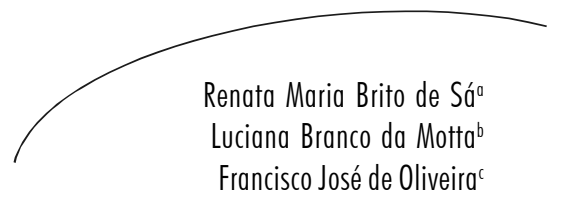

\section{RESUMO}

Insônia é um problema comum em todos os estágios da vida, mas é particularmente comum após os 65 anos de idade. É definida como uma dificuldade para iniciar o sono ou para se manter dormindo. Os distúrbios do sono nos idosos são comuns e multifatoriais. Vários fatores, incluindo idade avançada, influências psicossociais, doenças clínicas e psiquiátricas e uso de medicações podem estar associados com insônia. Apesar disso, os fatores de risco envolvidos no desenvolvimento de insônia não têm sido completamente identificados. A privação do sono interfere de maneira negativa na qualidade de vida. $\mathrm{O}$ objetivo deste trabalho é verificar a prevalência de insônia e de fatores de risco relacionados a esta queixa nos idosos acolhidos no ambulatório de geriatria do NAI (Núcleo de Atenção ao Idoso) da UERJ, em 2005, com 60 anos ou mais, de ambos os sexos. A busca ativa de insônia e sua correta avaliação são passos fundamentais na avaliação geriátrica.

\section{ABSTRACT}

Insomnia is a problem in all stages of life but is particularly common after age 65. It is defined as the inability to initiate or maintain sleep. Sleep disorders in the elderly are common and multifactorial. Several factors, including advanced age, psychosocial influences, clinical and psychiatric illnesses and the use of medications can be associates with insomnia. However, the risk factors involved in the insomnia development have not been completely identified. The privation
Palavras-chave: distúrbios do início e da manutenção do sono; idoso; transtornos do sono; prevalência; qualidade de vida; fatores de risco; avaliação geriátrica

Correspondência / Correspondence

Renata Maria Brito de Sá

Av. Cel. Miguel Sátyro, 200/2601 - Cabo Branco

58045-10 - João Pessoal, PB, Brasil

E-mail: re.brito@globo.com 
of sleep may impair the quality of life and daytime functioning. The objective of this work is verify the prevalence of insomnia and risk factors to this complaint in geriatric outpatients of NAI (Núcleo de Atenção ao Idoso- Elderly Care Center)- UERJ, in 2005, aged 60 years or more, of both sexes. A complete assessment of insomnia and its correct evaluation are basic steps in the geriatric evaluation.
Key words: sleep initiation and maintenance disorders; aged; sleep disorders; prevalence; quality of life; risk factors; geriatric assessment

\section{INTRODUÇÃO}

Desordens do sono são comuns e multifatoriais. ${ }^{3} \mathrm{O}$ sono é restaurador e, potencialmente, necessário à vida. Aproximadamente um terço da população geral apresenta alguma dificuldade para dormir. O risco de desordens do sono aumenta com a idade. ${ }^{21}$ Dentre essas desordens, insônia é a mais prevalente. ${ }^{6}$

Insônia é definida como uma dificuldade para iniciar o sono ou para se manter dormindo, quando pode haver uma diminuição total ou parcial da quantidade e/ou da qualidade do sono. Pode ser classificada em inicial, intermediária ou final, e, quanto à duração, em transitória ( $<1$ mês), de curto tempo (1 6 meses) ou crônica ( $>6$ meses). Também pode ser primária ou secundária a algum fator conhecido. ${ }^{31}$

Entre as desordens primárias, destacamse: a apnéia do sono e a síndrome das pernas inquietas, patologias que também atingem a população idosa. Apnéia do sono é uma condição definida por paradas repetidas e temporárias da respiração durante o sono, por obstrução das vias aéreas. Este distúrbio freqüentemente é associado com pacientes obesos e que roncam, com queixa de sonolência diurna excessiva, sono não reparador, despertares freqüentes durante a noite, cefaléia matutina e irritabilidade. ${ }^{27} \mathrm{~A}$ síndrome das pernas inquietas é uma desordem neurológica associada a sensações anormais nas pernas, uma irresistível necessidade de movimentar os membros inferiores, acompanhada de sensações de arrastamento das pernas. Estes sintomas causam despertares noturnos, que resultam na redução no período de sono e sonolência diurna. Cerca de 5 a $10 \%$ da população geral é acometida por este problema e a prevalência aumenta com o avançar da idade, chegando a atingir $10 \%$ das pessoas acima de 65 anos. $^{1,11}$

Insônia pode ser secundária a causas situacionais, como nos processos de institucionalização, internação hospitalar, viagens ou locais estranhos, onde o idoso, nessas situações, pode ter grande dificuldade de adaptação inicial, por se tratar de um ambiente estranho, às vezes barulhento, com outros horários e rotina. ${ }^{9}$ Também pode ser conseqüência de efeitos colaterais de alguns fármacos, como: anti-hipertensivos (metildopa, clonidina, reserpina, propranolol, atenolol e pindolol), anticolinérgicos (brometo de ipratrópio), broncodilatadores (terbutalina, salmeterol), 
xantinas (teofilina) e antidepressivos inibidores da recaptação da serotonina. Importante frisar que os anti-histamínicos e tranqüilizantes diazepínicos, muitas vezes usados para tratar insônia, podem causar efeito paradoxal em idosos. ${ }^{2,7}$ Substâncias como o álcool, nicotina e cafeína, consideradas estimulantes do sistema nervoso central, podem afetar a arquitetura normal do sono. ${ }^{31}$ Insônia também pode ser secundária a várias condições clínicas, sobretudo as crônicas. Quanto maior for o número de doenças clínicas associadas, maior a probabilidade de insônia. ${ }^{6}$ Entre essas doenças clínicas, consideradas fatores de risco para insônia, destacam-se: doenças genito-urinárias (hiperplasia prostática benigna e incontinência urinária), doenças gastrointestinais (doença do refluxo gastroesofágico e úlceras gastroduodenais), doenças cardiovasculares (insuficiência cardíaca descompensada e doença vascular periférica arterial ou venosa), doenças respiratórias crônicas, doenças metabólicas (diabetes, obesidade e hipertireoidismo) e doenças osteomioarticulares (osteoartrose, osteoporose e tendinites). ${ }^{17}$ É clássica também a associação de insônia com doenças psiquiátricas. As três situações psiquiátricas mais associadas à insônia no idoso são ansiedade, depressão e demência. ${ }^{29}$

É um problema comum em todos os estágios da vida, mas é particularmente importante após os 65 anos de idade. Estima-se que os transtornos do sono afetam em torno de $50 \%$ dos idosos e que, entre esses transtornos, a insônia tem prevalência de 20 a 40\% nessa população. ${ }^{2}$ Tem sido demonstrado também que insônia na velhice é mais prevalente em mulheres do que em homens. ${ }^{33}$
Em trabalho publicado pelo National Institute on Aging (NIA), com 9.000 indivíduos com 65 anos ou mais, apenas 12\% não relataram alguma dificuldade para dormir. As queixas de sono apresentadas foram dificuldade para iniciar o sono (43\%), despertares noturnos (30\%), cochilos diurnos (25\%), despertar muito cedo $(19 \%)$ e sono não restaurador $(13 \%){ }^{13}$

Insônia é hoje um problema de saúde pública e não é apenas importante por ser um problema comum, mas, sobretudo, por ser uma causa de fragilidade na população idosa, estando relacionada com grande prejuízo clínico e funcional. ${ }^{2,17} \mathrm{Na}$ maioria dos trabalhos epidemiológicos, insônia tem sido relacionada com uso freqüente dos recursos médicos, problemas crônicos de saúde e aumento no uso de medicamentos (polifarmácia). ${ }^{30} \mathrm{~A}$ privação do sono interfere de maneira negativa na qualidade de vida, diminuindo a capacidade de concentração, atenção e memória, favorece o desequilíbrio, aumenta a sonolência diurna, o que aumenta o risco de quedas e acidentes, todos preditores de maior morbimortalidade entre os idosos. ${ }^{22}$ Esses pacientes tendem a fazer uso indiscriminado de medicações conhecidas como sedativos hipnóticos, como os benzodiazepínicos, que induzem tolerância e dependência e aumentam ainda mais o risco de quedas. Idosos que sofrem de insônia têm uma probabilidade quatro vezes maior de sofrer uma queda, a qual pode resultar em ferimentos e fraturas. ${ }^{19,25}$

O padrão normal do sono muda com o avançar da idade. Mudanças sociais e fisiológicas do envelhecimento contribuem para tal fato. Mudança do padrão social, dos padrões 
familiares, diminuição do ciclo de amizades, inatividade física e profissional favorecem maior sonolência diurna, conseqüentemente, redução do sono noturno. ${ }^{26}$

Através de avaliações em laboratórios do sono, sabe-se que o tempo total de sono de pessoas idosas, de um modo geral, é menor do que o de adultos jovens. A quantidade de sono necessária para que se fique alerta no dia seguinte varia de pessoa para pessoa. Um recém-nascido dorme, em média, 16 horas. Um adulto jovem, cerca de 8 horas. Com o avançar da idade, essa duração é reduzida a cerca de 6 horas. $^{7}$ Não somente a duração, mas a continuidade do sono também é importante. Entre os idosos, é comum a ocorrência de sono interrompido e fragmentado. Há também uma diminuição da porcentagem do sono REM, menor tempo dos estágios 3 e 4 do sono NREM (sono profundo e restaurador), maior fragmentação do sono (despertares noturnos) e sonolência diurna. A latência do sono é encurtada durante o dia, levando a cochilos diurnos ocasionais ou freqüentes. ${ }^{3}$

Apesar da alta prevalência e das implicações negativas sobre a qualidade de vida desses pacientes, a insônia ainda tem sido inadequadamente diagnosticada e tratada, e os diversos fatores de risco envolvidos no seu desenvolvimento não têm sido completamente identificados. Inatividade progressiva, insatisfação com a vida social e presença de doenças clínicas e psiquiátricas podem ser os maiores preditores de insônia na população idosa. ${ }^{17}$

É importante diferenciar o normal do patológico quando se fala em insônia, avaliar e conhecer as mudanças que surgem com a idade, saber obter informações relacionadas com os distúrbios do sono e conhecer as condições patológicas mais comuns relacionadas à insônia, para que o tratamento seja realmente efetivo. $\mathrm{O}$ uso de questionários direcionados para esse problema ajuda a caracterizar melhor a queixa. O diário do sono também deve ter seu foco direcionado para identificar causas primárias e/ou secundárias. ${ }^{8} \mathrm{~A}$ busca ativa de insônia e sua correta avaliação são passos fundamentais na avaliação geriátrica.

O objetivo deste trabalho foi verificar a prevalência de insônia e de fatores de risco relacionados a essa queixa em uma população de idosos atendidos num ambulatório de geriatria.

\section{METODOLOGIA}

A população estudada foi composta dos idosos acolhidos no ambulatório de geriatria do NAI (Núcleo de Atenção ao Idoso) da Universidade Estadual do Rio de Janeiro em 2005, com 60 anos ou mais, de ambos os sexos. Foi feita uma revisão do prontuário desses pacientes, dividindo-os em dois grupos: $\mathrm{pa}-$ cientes com queixa de insônia (grupo 1) e pacientes sem queixa de insônia (grupo 2).

Foram analisadas, nos dois grupos, as seguintes variáveis: sexo, idade, estado civil, diagnósticos pré-estabelecidos de acordo com a Classificação Internacional de Doenças (CID-10) e medicações de uso regular. Foram investigados também nos dois grupos, as seguintes variáveis clínicas, consideradas 
como fatores de risco relacionados com insônia: depressão maior, ansiedade, demência, incontinência urinária, prostatismo, doença pulmonar obstrutiva crônica (DPOC), insuficiência cardíaca (IC), doença vascular periférica, úlceras gástrica ou duodenal, diabetes melitus (DM), hipertireoidismo, osteoartrose (AO), osteoporose, dor crônica, doença do refluxo gástrico esofágico (DRGE) e obesidade (diagnósticos registrados no prontuário pelo médico assistente de cada paciente, de acordo com o CID -10).

Os dados foram estratificados e armazenados em um banco de dados (Microsoft Excel 2002), com o objetivo de verificar a prevalência de insônia na população em estudo e avaliar a associação desta queixa com os fatores de risco através do teste do Qui-qua- drado $\left(X^{2}\right)$. O nível de significância considerado foi $\mathrm{p}<0,05$.

Os pacientes do grupo 1 foram entrevistados individualmente. Estas entrevistas foram agendadas por contato telefônico ou pessoalmente com os sujeitos, conforme a sua disponibilidade. A entrevista foi aplicada pelo próprio pesquisador, após a exposição dos riscos e benefícios envolvidos na presente pesquisa disposta no Termo de Consentimento Livre e Esclarecido. Foi aplicado um questionário estruturado sobre o tema para avaliação do padrão e da qualidade do sono do paciente. Após a entrevista, foi distribuído folheto informativo contendo orientações sobre higiene do sono (quadro 1), visando melhorar a queixa de insônia.

Quadro 1 - Higiene do Sono

\section{Horário regular para deitar e levantar}

É importante respeitar esses horários, inclusive nos finais de semana, pois favorece o funcionamento do relógio biológico. Mudanças de hábito podem atrapalhar o sono.

\section{Preparação do ambiente}

Calor e frio excessivos alteram bastante o sono, portanto tentar manter o quarto com temperatura agradável.

Ruídos podem ser a causa de um sono ruim.

Quando houver:

muita luz: escureça o ambiente;

muito barulho: elimine ou reduza o barulho;

\section{O que se deve comer antes de dormir}

Procurar fazer refeições mais leves antes de deitar e não deitar logo após alimentar-se. O ideal é aguardar cerca de 1 hora. 
Quadro 1 - Higiene do Sono (continuação)

\section{O que não se deve beber antes de dormir}

Não é aconselhado tomar líquido imediatamente antes de dormir, pois haverá um aumento do estímulo de diurese, fazendo levantar mais vezes à noite para ir ao banheiro. Não se deve beber café, chá preto, mate, refrigerantes após as 17 horas, pois estas bebidas possuem substâncias estimulantes que dificultam o sono.

As bebidas alcoólicas, embora ajudem a relaxar, perturbam a qualidade do sono. Pessoas que roncam devem evitá-las, pois pode haver piora do ronco e das pausas respiratórias, devido ao relaxamento provocado pelo álcool na musculatura respiratória.

\section{Não ler ou ver TV na cama}

Quem tem insônia deve evitar ler e assistir à televisão antes de dormir. O quarto de dormir não deve ser utilizado para trabalhar, estudar ou comer.

\section{Não fume antes de dormir}

A nicotina favorece a insônia e a um sono não reparador.

\section{Pratique esportes}

Faça atividades físicas regularmente, porém evite exercícios fortes no final do dia. Este tipo de exercício deve ser feito no período da manhã. No final do dia, os exercícios devem ser mais leves como alongamentos ou caminhadas, e, pelo menos, 4 horas antes de dormir. Procure seu médico antes de iniciar o seu programa de exercícios.

\section{Relaxe}

Procurar relaxar o corpo e a mente antes de ir para a cama. Nunca tentar resolver problemas antes de dormir.

Todas as fases deste trabalho seguiram as exigências éticas e científicas contidas na resolução 196/96 do Conselho Nacional de Saúde (CNS) e seu início se deu após avaliação e aprovação pelo Comitê de Ética em Pesquisa da Universidade Estadual do Rio de Janeiro (parecer COEP 061/2006).

\section{RESULTADOS}

Dos 116 pacientes inseridos no Núcleo de Atenção ao Idoso da UERJ em 2005, 11 não participaram da pesquisa (2 óbitos e 9 sem comunicação), ficando nossa população em estudo com um total de 105 idosos. Destes, 76 $(72,38 \%)$ eram do sexo feminino e $29(27,62 \%)$ do sexo masculino, com idades variando entre 60 e 91 anos e média de 73,2 anos (tabela 1). 
Tabela 1 - Características sociodemográficas dos pacientes com e sem insônia.

\begin{tabular}{|c|c|c|c|}
\hline Variável & $\begin{array}{c}\text { Total } \\
n=105\end{array}$ & $\begin{array}{c}\text { Com insônia } \\
n=34\end{array}$ & $\begin{array}{c}\text { Sem insônia } \\
n=71\end{array}$ \\
\hline \multicolumn{4}{|l|}{ Sexo } \\
\hline Feminino & 76 & 27 & 49 \\
\hline Masculino & 29 & 7 & 22 \\
\hline \multicolumn{4}{|l|}{ Faixa etária } \\
\hline $60-69$ anos & 36 & 9 & 27 \\
\hline $70-84$ anos & 67 & 25 & 42 \\
\hline$>85$ anos & 2 & 0 & 2 \\
\hline
\end{tabular}

A prevalência de insônia, representada no gráfico 1, foi 32,38\% (34 idosos). Houve prevalência maior de insônia no sexo feminino $(79,41 \%)$. Entre os pacientes com insônia, 33 $(97,03 \%)$ convivem com esse problema há mais de 6 meses (insônia crônica), 27 (79,41\%) deles relataram insônia inicial e 22 (64,71\%) intermediária e final. O tempo total de sono foi em média de 4,07 horas por noite. Mais da metade dos insones $(61,76 \%)$ relatou cochilos diurnos, que duravam em média 0,8 horas. A queixa de cansaço diurno intenso atingiu cerca de $85 \%$ dos pacientes com insônia e
$73,53 \%$ responderam que a dificuldade para dormir interfere muito nas suas atividades de vida diária, no humor, memória, concentração e nas funções do trabalho. Usam algum tipo de ansiolítico 19 pacientes (55,88\%) e, entre os que não fazem uso desse tipo de medicação, 7 (46,67\%) já usaram em algum outro momento. Dos 34 idosos com insônia, $3(8,82 \%)$ são fumantes, $7(20,59 \%)$ consomem mais de três xícaras de café por dia e nenhum deles tem o hábito de consumir algum tipo de bebida alcoólica para melhorar o padrão do sono.

Gráfico 1- Prevalência de insônia na população total em estudo

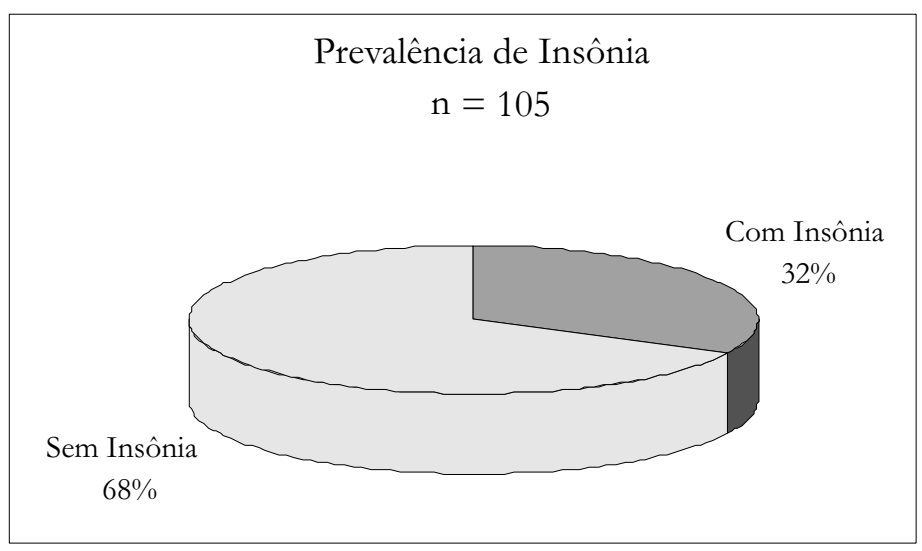


De acordo com a tabela 2, depressão, ansiedade, incontinência urinária e prostatismo, osteoartrose, doença vascular periférica, demência, osteoporose, DPOC, dor crônica e DRGE foram os fatores de risco relacionados à queixa de insônia com signi- ficância estatística $(p<0,05)$. Não houve diferença estatística entre os dois grupos em relação a úlceras gástricas e duodenais, diabetes, obesidade e ICC. Não foi evidenciado nenhum caso de hipertireoidismo na população em estudo.

Tabela 2 - Associação entre os fatores de risco e a queixa de insônia

\begin{tabular}{|c|c|c|c|c|c|}
\hline $\begin{array}{l}\text { FATORES DE RISCO } \\
\text { (FR) }\end{array}$ & $\begin{array}{c}\text { Insônia }+ \\
\text { FR }+\end{array}$ & $\begin{array}{l}\text { Insônia }+ \\
\text { FR - }\end{array}$ & $\begin{array}{c}\text { Insônia - } \\
\text { FR + }\end{array}$ & $\begin{array}{l}\text { Insônia - } \\
\text { FR - }\end{array}$ & $P^{*}$ \\
\hline DEPRESSÃO & 19 & 15 & 7 & 64 & $p<0,001$ \\
\hline ANSIEDADE & 22 & 12 & 2 & 69 & $p<0,001$ \\
\hline I. U. ${ }^{1 / P R O S T A T I S M O}$ & 22 & 12 & 24 & 47 & $p<0,01$ \\
\hline OSTEOARTROSE & 25 & 9 & 24 & 47 & $p<0,001$ \\
\hline $\begin{array}{l}\text { ÚLCERAS } \\
\text { GÁSTRODUODENAIS }\end{array}$ & 3 & 31 & 3 & 68 & $p>0,05$ \\
\hline DÇ. VASC. PERIF. 2 & 13 & 21 & 12 & 59 & $p<0,05$ \\
\hline DEMENCIA & 9 & 25 & 6 & 65 & $p<0,05$ \\
\hline DIABETES & 10 & 24 & 16 & 55 & $p>0,05$ \\
\hline OBESIDADE & 5 & 29 & 13 & 58 & $p>0,05$ \\
\hline OSTEOPOROSE & 14 & 20 & 7 & 64 & $p<0,001$ \\
\hline $\mathrm{DPOC}^{3}$ & 6 & 28 & 2 & 69 & $p<0,025$ \\
\hline DOR CRÔNICA & 27 & 7 & 8 & 63 & $p<0,001$ \\
\hline $\mathrm{DRGE}^{4}$ & 9 & 25 & 4 & 67 & $p<0,01$ \\
\hline $\mathrm{ICC}^{5}$ & 5 & 29 & 4 & 67 & $p>0,05$ \\
\hline
\end{tabular}

* Nível de significância: valor de $p<0,05$

${ }^{1}$ I.U.: Incontinência urinária; ${ }^{2}$ Dç. Vasc. Perif.: Doença vascular periférica; ${ }^{3} \mathrm{DPOC}$ : Doença pulmonar obstrutiva crônica; ${ }^{4}$ DRGE: Doença do refluxo gastroesofágico; ${ }^{5}$ ICC: Insuficiência cardíaca congestiva

Nota: em negrito, fatores de risco com significância estatística 
O grupo de pacientes com insônia apresentou, em média, 5,56 fatores de risco. Já o grupo sem insônia apresentou uma média de apenas 1,86 fatores de risco.

\section{DISCUSSÃO}

Os distúrbios do sono constituem um significante problema de saúde pública. $\mathrm{O}$ padrão normal do sono muda com o avançar da idade. Mudanças sociais e fisiológicas do envelhecimento contribuem para tal fato. ${ }^{9}$

Estudos epidemiológicos retratam uma alta prevalência de insônia, principalmente na população idosa. Essa prevalência varia entre 5 e $35 \%$, dependendo da metodologia utilizada e da amostra selecionada. Quanto mais idosa a população em estudo, mais alta tende a ser a prevalência de insônia, o que justifica a alta prevalência $(32,38 \%)$ encontrada em nosso trabalho.

As mudanças hormonais ocorridas no sexo feminino no período pós-menopausa têm sido a explicação para a maior prevalência de insônia encontrada entre as mulheres. Essa diferença tende a ser menor quando a população estudada é mais jovem. ${ }^{33}$

O uso de um questionário estruturado para avaliar a queixa de insônia é uma ferramenta importante, que auxilia o profissional de saúde na identificação de possíveis causas para o problema, ajudando a determinar a dimensão e o impacto sobre as funções de vida diária do paciente e colaborando para uma melhor caracterização da insônia. ${ }^{29} \mathrm{O}$ modelo proposto neste trabalho pode ser utilizado para tal fim.

É importante classificar a insônia quanto a sua duração. Observamos que quase todos os pacientes com insônia apresentavam esse problema há mais de 6 meses, provavelmente porque, como esses pacientes foram inseridos no nosso ambulatório em 2005 e nossa pesquisa se desenvolveu em 2006, perdemos, nesse intervalo, possíveis casos de insônia transitória e de curta duração. Apesar disso, é relativamente comum uma maior prevalência de insônia crônica, principalmente quando se trata de uma população mais idosa. A prevalência de insônia ocasional não tende a mudar com o avançar da idade. ${ }^{4}$

Fisiologicamente, há uma diminuição do tempo total do sono com o envelhecimento, com uma duração média de 6 horas, nas 24 horas do dia. Em nossa amostra, o tempo total de sono dos pacientes com insônia foi reduzido a 4,07 horas. Essa redução resulta em insatisfação, pois não corresponde a um tempo suficiente para um sono restaurador.

Insônia não é apenas uma diminuição da quantidade de horas de sono, mas também uma piora da qualidade das horas dormidas. A avaliação do grau de insatisfação com a qualidade do sono deve ser questionada a todo paciente com insônia. Através do questionário aplicado, pode-se perceber o impacto negativo deste problema na qualidade de vida desses idosos.

Os cochilos diurnos podem fazer parte do problema, ao reduzirem a urgência de dor- 
mir no horário convencional ou serem conseqüência de uma noite mal dormida. Mais da metade dos insones relatou cochilos diurnos, o que pode ter influência negativa na vida social desses pacientes e aumentar o risco de acidentes, como quedas. ${ }^{3}$

Insônia pode ser considerada uma condição primária, mas pode coexistir com outras desordens ou ser considerada secundária a essas desordens. A maioria dos idosos sofre de uma ou mais doenças crônicas. Como descrito na literatura, várias dessas condições clínicas são consideradas fatores de risco importantes para o desenvolvimento de insônia. ${ }^{28}$ Deve-se sempre tentar determinar a causa de insônia, sabendo que ela pode estar associada a mais de um fator. ${ }^{21}$ No nosso estudo, foram considerados fatores de risco com significância estatística $(p<0,05)$ : depressão, transtorno da ansiedade, incontinência urinária e prostatismo, osteoartrose, doença vascular periférica, demência, osteoporose, DPOC, dor crônica e DRGE. O grupo com insônia apresentou uma média de 5,56 fatores de riscos, três vezes mais que o grupo sem insônia, o que revela seu caráter multifatorial.

O estado de tensão produzido por estresse, embora seja encontrado em qualquer idade, tem prevalência muito acentuada nos idosos. As três situações psiquiátricas mais associadas à insônia no idoso são depressão, ansiedade e demência. Mais de 90\% dos quadros depressivos cursam com mudanças no ciclo sonovigília, e cerca de $50 \%$ deles são acompanhados de insônia. ${ }^{10}$ Morte de amigos, perda do cônjuge, perda do espaço social, dificuldades financeiras, sentimentos de abandono por par- te da família, limitações físicas próprias da idade, mudanças no status social, percepção da própria condição de saúde, vários são os motivos de alterações depressivas no idoso. A insônia nesses pacientes pode ser tanto inicial, intermediária ou terminal. Normalmente, salvo muitas exceções, a ansiedade é mais responsável por insônia inicial, ou dificuldade para conciliar o sono, e à insônia intermediária, proporcionando o ato de levantar no meio da noite e não conseguir dormir mais. É comum a associação de quadros depressivos com transtornos da ansiedade, e quem tem insônia tem probabilidade muito maior de desenvolver quadros de depressão e ansiedade. ${ }^{32}$ As síndromes demenciais também se apresentam comumente com desordens do sono, tanto na sua evolução natural, pela piora gradual da dependência funcional, como também por outros problemas apresentados por esses pacientes, como os distúrbios de comportamento. O diagnóstico de insônia em pacientes demenciados é de difícil caracterização pela anamnese, sendo os familiares e os cuidadores fundamentais para que se obtenha o maior número de dados. Este sintoma deve ser abordado de forma cuidadosa, e fatores desencadeantes que contribuem para a alteração do ciclo sono-vigília devem ser pesquisados, tais como: estado confusional agudo, desidratação, distúrbios eletrolíticos, infecções, constipação intestinal, dor crônica, cardiopatias. Na demência, ainda em seus estágios iniciais, pode haver inversão do ciclo circadiano, quando o paciente troca o dia pela noite. Esta inversão tem sido uma das principais causas de internação em instituições de longa permanência. ${ }^{10} \mathrm{~A}$ insônia nesses casos tem 
forte impacto negativo na qualidade de vida tanto dos pacientes quanto de seus cuidadores.

Doenças genito-urinárias, como hiperplasia prostática benigna e incontinência urinária, podem levar a urgência urinária e despertares noturnos freqüentes, fazendo com que o paciente se levante várias vezes à noite para urinar. ${ }^{28}$

Doenças gastrointestinais, como a doença do refluxo gastroesofágico, cursa com tosse crônica e pirose noturna. As úlceras duodenais podem despertar o paciente à noite com uma dor intensa, conhecida como "clocking". As úlceras gástricas levam à dor crônica em queimação, também perturbando o sono. ${ }^{28}$

Pacientes com doenças cardiovasculares também podem-se apresentar com insônia por interferência direta dos sintomas clínicos dessas doenças. Doença vascular periférica, arterial ou venosa, pode cursar com quadro álgico importante, dormência, câimbras e aumento da diurese noturna.

Entre as doenças respiratórias, a doença pulmonar obstrutiva crônica está muito relacionada com a piora da qualidade do sono, por interferir diretamente no processo da respiração e por produzir, freqüentemente, dispnéia e tosse crônicas. ${ }^{20}$

Doenças osteomioarticulares, como osteoartrose e osteoporose, prejudicam o ciclo do sono, principalmente por dor crônica, esforços posturais e dificuldade de acomodação. ${ }^{20,23}$

Quadros de dor crônica, decorrentes, por exemplo, de doenças oncológicas, cursam freqüentemente com depressão e ansiedade.
A insuficiência cardíaca descompensada leva a dispnéia paroxística noturna, tosse e maior diurese noturna, pela reabsorção dos edemas. O diabetes, doença metabólica muito prevalente na população, quando descompensado, pode-se apresentar com problemas urinários, que aumentam a freqüência urinária, despertando o paciente à noite. Complicações crônicas do diabetes, como neuropatia periférica, cursam com processos dolorosos que interferem diretamente na qualidade do sono. ${ }^{28}$ Esses dois fatores não tiveram significância estatística no nosso trabalho, provavelmente porque os pacientes da nossa população se apresentavam clinicamente compensados desses problemas no momento da avaliação.

O álcool pode afetar a arquitetura normal do sono. Muitos fazem uso do álcool para melhorar a insônia. Quando o álcool é usado como hipnótico, ele pode inicialmente diminuir o tempo de latência do sono, mas produz, no decorrer da noite, uma maior fragmentação e diminuição do sono REM. ${ }^{31}$ Não foi encontrado nenhum caso de uso do álcool para esse fim em nosso trabalho. Substâncias estimulantes, como nicotina e cafeína, são notoriamente causadoras de insônia e deveriam ser evitadas. Cafeína, muito consumida na população em geral, é associada com aumento do período de latência do sono, redução da eficiência do sono e despertares espontâneos. ${ }^{22}$ Consumiam uma quantidade relativamente grande de cafeína por dia (mais de três xícaras de café), 20,59\% dos insones, e 8,82\% destes eram fumantes. $\mathrm{O}$ uso dessas substâncias deveria ser desaconselhado para os indivíduos que sofrem de insônia. ${ }^{14}$ 
Pacientes com insônia tendem a fazer uso indiscriminado de medicações conhecidas como sedativos hipnóticos, como os benzodiazepínicos. Observamos uma alta prevalência do uso desses fármacos $(55,88 \%)$ e, entre os que não estavam fazendo uso desse tipo de medicação, 46,67\% já usaram em algum outro momento. Essas medicações, quando usadas cronicamente, induzem tolerância e dependência, aumentam o risco de quedas, pioram a apnéia do sono, diminuem a coordenação motora, levam a comprometimento da função mental, confusão e amnésia anterógrada, além de não conseguirem resolver o problema de insônia no longo prazo. ${ }^{15}$

As orientações sobre higiene do sono, oferecidas a todos os nossos pacientes insones, são consideradas estratégias efetivas no processo de intervenção não-farmacológica da insônia. ${ }^{5}$

\section{CONCLUSÃO}

Podemos concluir que há uma alta prevalência de insônia crônica e secundária entre os idosos; que a privação do sono interfere de maneira negativa na qualidade de vida dessa população; e que o sucesso na avaliação e no tratamento da insônia depende da busca ativa dessa queixa e do conhecimento e correção de todos os fatores de risco relacionados ao problema, como depressão, transtorno da ansiedade, incontinência urinária e prostatismo, osteoartrose, doença vascular periférica, demência, osteoporose, DPOC, dor crônica e DRGE.

\section{NOTAS}

Residência médica em Geriatria e Gerontologia, HUPE, Universidade do Estado do Rio de Janeiro, Rio de Janeiro, RJ, Brasil.

b,c Médicos, mestres em Saúde Coletiva, Universidade do Estado do Rio de Janeiro, Rio de Janeiro, RJ, Brasil. E-mails: lumotta@uerj.br e fjoliveira@superig.com.br

Artigo resultado de pesquisa premiada em $2^{\circ}$ lugar no $3^{\circ}$ Prêmio de Residência Médica do Conselho Regional de Medicina do Rio de Janeiro - Cremerj, em dezembro/ 2006.

\section{AGRADECIMENTOS}

A todos da equipe NAI (Núcleo de Atenção ao Idoso da UERJ), que, direta ou indiretamente, contribuíram para realização deste trabalho.

\section{REFERÊNCIAS}

1. Allen RP, Earley CJ. Restless legs syndrome: a review of clinical and pathophysiologic features. J Clin Neurophysiol 2001; 18:128-47.

2. Ancoli-Israel S, Cooke JR. Prevalence and comorbidity of insomnia and effect on functioning in elderly populations. J Am Geriatr Soc 2005 Jul;53(7 Suppl):S264-71.

3. Avidan AY. Sleep disorders in the older patient. Prim Care 2005 Jun;32(2):563-86.

4. Ayalon L, Liu L, Ancoli-Israel S. Diagnosing and treating sleep disorders in the older adult. Med Clin North Am 2004 May;88(3):737-50, ix-x.

5. Bain KT. Management of chronic insomnia in elderly persons. Am J Geriatr Pharmacother 2006 Jun;4(2):168-92.

6. Benka RM. Diagnosis and treatment of chronic insomnia: a review. Psychiatr Serv 2005; 56(3):332-43. 
7. Camara VD, Camara WS. Distúrbios do sono no idoso. In: Freitas E, et al, organizador. Tratado de Geriatria e Gerontologia. Rio de Janeiro: Guanabara Koogan; 2002. p.189-95.

8. Chesson A, Hartse K, Anderson WM, Davila D, Johnson S, Littner M, Wise M, Rafecas J. Practice parameters for the evaluation of chronic insomnia. An American Academy of Sleep Medicine report. Standards of Practice Committee of the American Academy of Sleep Medicine. Sleep. 2000 Mar 15;23(2):237-41.

9. Coll PP. Distúrbios do sono. In: Adelamn AM. Geriatria : 20 problemas + comuns. Rio de Janeiro: Revinter, c2004. p.173-88.

10. Cricco M, Simonsick EM, Foley DF. The Impact of insomnia on cognitive functioning in older adults. J Am Geriatr Soc 2001; 49(9):1185-1189.

11. Earley CJ. Restless Legs Syndrome. The New England journal of medicine 2003; 348(21):2103-9.

12. Foley DJ, Ancoli-Israel S, Britz P, Walsh J. Sleep disturbances and chronic disease in older adults: results of the 2003 National Sleep Foundation Sleep in América Survey. J Psychosom Res 2004; 56(5):497-502.

13. Foley DJ, et al. Sleep complaints among elderly persons: an epidemiologic study of three communities. Sleep 1995; 18(6):425-32.

14. Gillin JC, Byerley WF. The diagnosis and management of insomnia. The New England journal of medicine 1990; 322(4):239-48.

15. Hauri PJ. Cognitive deficits in insomnia patients. Acta Neurol Belg 1997; 97(2):113-7.

16. Jao DV, Alessi CA. Sleep disorders. In: Landerfeld CS, et al, editor. Current geriatric diagnosis \& treatment. USA: McGraw-Hill; 2004. p.114-21.
17. Kamel NS, Gammack JK. Insomnia in the elderly: cause, approach, and treatment. Am J Med 2006; 119(6):463-9.

18. Klink ME, Quan SF, Kaltenborn WT, Lebowitz MD. Risk factors associated with complaints of insomnia in a general adult population. Influence of previous complaints of insomnia. Arch Intern Med 1992;152(8):1634-7.

19. Koski K, Luukinen H, Laippala P, Kivela SL. Risk factors for major injurious falls among the home-dwelling elderly by functional abilities. A prospective population-based study. Gerontology 1998; 44:232-8.

20. Krystal AD. The Effect of Insomnia Definitions, Terminology, and Classifications on Clinical Practice. J Am Geriatr Soc 2005; 53(7):258-263.

21. Kupfer DJ, Reynolds, CF. Management of Insomnia. The New England journal of medicine 1997; 336(5):341-6.

22. McCall WV. Diagnosis and Management of Insomnia in Older People. J Am Geriatr Soc 2005; 53(7):272-77.

23. Monane M. Insomnia in the elderly. J Clin Psychiatry 1992; 53(6):23-8.

24. Moran MG, Stoudemire A. Sleep disorders in the medically ill patient. J Clin Psychiatry 1992; 53(6):29-36.

25. Phillips B, Mannino DM. Does insomnia kill? Sleep. 2005; 28(8):965-71.

26. Prinz PN, Vitiello MV, Raskind MA, Thorpy MJ. Geriatrics: sleep disorders and aging. The New England journal of medicine 1990; 323(8):520-6.

27. Razen JE. Sleep apnea syndrome. The New England journal of medicine 2002; 346(6):404-12.

28. Sateia MJ, Nowell PD. Insomnia. Lancet 2004; 364(27):1959-73. 
29. Schneider DL. Insomnia: safe and effective therapy for sleep problems in the older patient. Geriatrics 2002; 57(5):24-35.

30. Silber MH. Chronic insomnia. The New England journal of medicine 2005; 353(8):803-10.

31. Summers MO, Crisostomo MI, Stepanski EJ. Recent Developments in the classification, evaluation and treatment of insomnia. Chest 2006; 130(1):276-86.
32. Taylor DJ, et al. Epidemiology of insomnia, depression, and anxiety. Sleep 2005; 28(11):1457-64.

33. Zhang B, Wing YK. Sex differences in insomnia: a meta-analysis. Sleep 2006; 29(1):85-93.

Recebido: $02 / 2 / 2007$

Aceito: 30/4/2007 